\title{
Rehabilitation with Cochlear Implant in Patient with Harboyan Syndrome
}

\author{
Lauren Medeiros Paniagua ${ }^{1,2}$ Maria Elza Kazumi Yamaguti Dorfman ${ }^{3}$ Luiz Lavinsky, ${ }^{4,5}$ Pricila Sleifer ${ }^{6}$
}

\footnotetext{
${ }^{1}$ Doctor of Science in Children's and Teenager's Health, Federal University of Rio Grande do Sul-UFRGS, Porto Alegre/RS, Brazil

2 Professor of Speech-Language Pathology, Fatima's University (RS), Rio de Janeiro/RJ, Brazil

${ }^{3}$ Speech-Language Pathologist, Voice Specialist, Hospital de Clinicas de Porto Alegre, Porto Alegre/RS, Brazil

${ }^{4}$ Department of Otorhinolaryngology, University Hospital of Porto

Alegre, Porto Alegre/RS, Brazil

${ }^{5}$ Graduate Program in Medicine: Surgery, Medical School, Federal

University of Rio Grande do Sul, Porto Alegre/RS, Brazil

${ }^{6}$ Audiologist, Professor of Federal University of Rio Grande do Sul

(UFRGS), Porto Alegre/RS, Brazil
}

\author{
Address for correspondence Lauren Medeiros Paniagua, Avenida João \\ Wallig, 1705/627, 91340001, Porto Alegre/RS, Brazil \\ (e-mail: Imedeirospaniagua@yahoo.com.br).
}

Int Arch Otorhinolaryngol 2013;17:403-406.

\begin{abstract}
Keywords

- cochlear implants

- hearing loss

- sensorineural

- rehabilitation of speech and language disorders

Background Harboyan syndrome, defined as congenital corneal dystrophy associated with progressive sensorineural hearing loss, was first described by Harboyan in 1971. It is a hereditary disease manifested by eye lesions consistent with corneal endothelial dystrophy and progressive sensorineural hearing loss. There is bilateral symmetric progressive hearing loss, which may be either dominant or recessive.

Objective To report a case of a patient with a diagnosis of Harboyan syndrome.

Case Report A 25-year-old woman with profound bilateral sensorineural hearing loss, showing poor hearing performance while using a personal sound amplification device, underwent hearing rehabilitation with a cochlear implant.

Conclusion Rehabilitation was imperative in this case. The cochlear implant has proven to be the best therapeutic option, providing the patient with a better quality of life.
\end{abstract}

\section{Introduction}

Harboyan syndrome, defined as congenital corneal dystrophy associated with progressive sensorineural hearing loss, was first described by Harboyan et al in 1971. It is a hereditary disease manifested by eye lesions consistent with corneal endothelial dystrophy and progressive sensorineural hearing loss. ${ }^{1}$

There are two different types of congenital hereditary corneal dystrophies: dominant malformation, which seems to be related to traits of other constitutional anomalies, and recessive degeneration, which is the most common type of this disorder in families with consanguinity. ${ }^{2}$ The dominant variant affects only $12 \%$ of cases of corneal dystrophy, where- as $1 \%$ of cases are reported as $\mathrm{X}$-linked recessive inheritance disorder. $^{2}$

Patients usually have bilateral symmetric progressive hearing loss after birth, which is suggestive of an acquired disease. Cawthorne and Hinchcliffe suggested that hearing loss may occur at any time from birth to 30 years of age. The authors classified this type of disease as a heredodegenerative disorder. ${ }^{3}$

The association of congenital hereditary corneal dystrophy with progressive sensorineural hearing loss suggests the diagnosis of Harboyan syndrome. ${ }^{1}$

The scarcity of data related to this disease, especially regarding the degree of involvement of the auditory function, received

April 22, 2011

accepted

April 15, 2013
Copyright $\odot 2013$ by Thieme Publicações Dol http://dx.doi.org/ Ltda, Rio de Janeiro, Brazil

$10.1055 / \mathrm{s}-0033-1351669$. ISSN 1809-9777. 
prompted us to report the case of a patient diagnosed with Harboyan syndrome. This patient had profound bilateral sensorineural hearing loss and she showed poor hearing performance while using a hearing device. She underwent successful aural rehabilitation with a cochlear implant $(\mathrm{CI})$.

\section{Review of the Literature}

Hearing and vision are two major sensory modalities related to the development and achievement of successful communication. Several diseases that affect hearing and vision, such as Cogan syndrome and Harboyan syndrome, have been described in the literature. The latter is a rare inherited disease manifested by eye lesions and progressive sensorineural hearing loss. ${ }^{1,2}$

Patients with diseases that affect different sensory organs need to be followed by a multidisciplinary team with the purpose of prevention, diagnosis, and treatment. Thus, speech therapists and otolaryngologists should be members of such a team so that clinical and therapeutic effectiveness could be achieved to improve the patients' quality of life. .,4 $^{3}$

There is a scarcity of data on Harboyan syndrome, especially with regard to hearing impairment and possibility of rehabilitation. We could not find another report of a case of hearing loss due to Harboyan syndrome treated with $\mathrm{Cl}$ in the literature.

\section{Case Report}

A 25-year-old woman from Porto Alegre, Rio Grande do Sul, Brazil, had an uneventful gestational history and was born by vaginal delivery at term. Her 5-minute Apgar score was 9 . She was referred to the Hospital de Clínicas de Porto Alegre (HCPA) for progressive hearing loss. The patient reported that the onset of the disease occurred after 2 years of age, with decreased visual acuity. She started to show progressive hearing loss at the age of 10 years. Otolaryngologic examination showed normal findings on otoscopy. Physical examination of the head and neck also did not reveal any abnormalities.

Audiologic evaluation showed symmetrical profound bilateral sensorineural hearing loss, type A tympanometric curves, and absent acoustic reflexes in both ears. Additionally, we found no otoacoustic emissions in both ears and absence of response of brain stem auditory evoked potentials. Additional tests, including STORCH (syphilis, toxoplasmosis, rubella, cytomegalovirus, and herpes simplex), electroencephalogram, brain and temporal bone computed tomography scan, chromosome analysis, urine chromatography for mucopolysaccharidosis, and metabolic screening, did not show any changes. Given the patient's progressive hearing loss, she had a well-established oral language code.

After otolaryngologic and audiologic examination, according to the protocol of the Department of Otolaryngology of the HCPA, the patient underwent aural rehabilitation with the use of bilateral personal sound amplification device ( 10 hours a day) without success. In mid 2005, we performed a speech examination including communication, language, speech, and voice. ${ }^{10}$ In addition, auditory discrimination of vowels, disyllables, trisyllables, and sentences was checked. These sentences were evaluated using either lip-reading or not, based on visual support from gesture and facial expressions. Auditory discrimination was also evaluated using two different methods: open set (predefined speech activity) and closed set (predictable or predefined speech activity). The patient was able to communicate using oral language and the Brazilian Sign Language (Libras). She also demonstrated spontaneous vocabulary, normal speech, and adapted voice (typical of deaf individuals). Nevertheless, she failed all specific tests (discrimination of vowels, disyllables, trisyllables, and sentences) when there was no lip-reading (i.e., the patient could not understand what was being said without visual support from mime and facial expression).

Based on these data and because she demonstrated a wellestablished oral language code, the patient was considered a candidate for $\mathrm{CI}$; however, she refused such treatment. In 2006, the patient returned to the Department of Otolaryngology of the HCPA because she decided to undergo hearing rehabilitation according to the treatment proposed. She was then included in the multidisciplinary assessment protocol of the HCPA and the Care Program for Profound/Severe Deaf Patients. The multidisciplinary team consisted of speech therapists, otolaryngologists, psychologists, and social workers.

The surgery was performed at the HCPA on May 26, 2006. The procedure was uneventful, and the patient was implanted with a Nucleus $24 \mathrm{CI}$, ESPrit 3G processor in the left ear. Electrode activation was performed 45 days after the date of the surgery. Then, the patient underwent aural rehabilitation provided by the team of speech therapists. She was followed by the otolaryngologists according to the protocol of the institution. The patient did not have any complications during the follow-up period.

During preoperative audiologic procedures, the following results were found in both ears: (1) pure tone audiometry: profound bilateral sensorineural hearing loss; (2) speech audiometry: no voice detection threshold and speech recognition index of $0 \%$; (3) distortion product otoacoustic emissions: absent; (4) brainstem auditory evoked potential: absent; (5) measurements of acoustic impedance: type A tympanometric curves (according to Jerger classification, 1970) and absent acoustic reflexes. We detected proper electrode impedance and presence of response on neural telemetry of all electrodes tested during perioperative procedures. During postoperative audiologic procedures, we found the lowest and highest hearing thresholds of $30 \mathrm{~dB}$ and $45 \mathrm{~dB}$, respectively, using freefield audiometry.

Postoperative speech examination for communication showed the following results: communication was performed mainly by means of oral language and, less frequently, through sign language. The patient had spontaneous vocabulary, normal speech, adapted voice, and open set communication (i.e., without agreeing with the examiner on what to say). The patient showed improved hearing results, reaching $75 \%$ accuracy in sentences without using lip-reading in open 
set tasks and $100 \%$ accuracy in sentences without lip-reading in closed set tasks.

\section{Discussion}

Currently, $\mathrm{Cl}$ is an excellent option for aural rehabilitation of patients with profound bilateral sensorineural hearing loss, provided that they are eligible for the procedure. When the patient has more than one irreversible sensory deficit in addition to hearing loss, hearing rehabilitation becomes a priority. Puga et al described a progressive worsening of visual and hearing impairment, and sensorineural hearing loss may initially reach high frequencies. ${ }^{5}$ Chiari et al described a case study of Cogan's syndrome with associated deafness and blindness. ${ }^{4}$ The authors reported that professionals from diverse areas should classify individuals with simultaneous visual and hearing loss as "deafblind" people. Such condition cannot be considered a simple sum of sensory losses; instead, it is a multiplying factor by means of which the combination of sensory losses results in serious communication and development problems.

Differential diagnoses of the Harboyan syndrome are Hurler disease, congenital glaucoma, Cogan syndrome, and syphilitic interstitial keratitis. ${ }^{6}$ These disorders were ruled out by additional exams.

Inherited hearing loss can be either dominant or recessive, but the latter is the most prevalent type. The pathogenesis of progressive sensorineural hearing loss is not well established. Paparella et al in 1969 described familial progressive sensorineural hearing loss associated with histopathologic findings compatible with marked degeneration of the stria vascularis and reduced ganglion cells mainly in the basal cochlear turn. ${ }^{6,7}$ However, the audiologic evaluation of these patients usually reveals no recruitment and discreet presence of positive tone decay. These findings are consistent with a greater chance of lesions in the organ of Corti and the auditory nerve (peripheral lesion). Such lesions can be treated with hearing rehabilitation with $\mathrm{CI}$, which partially explains the success of this type of treatment in our patient.

\section{Conclusion}

As, to the best of our knowledge, this is the first case report of hearing loss caused by Harboyan syndrome treated with CI, it was not possible to perform a comparative analysis of our results with those of other authors. At the time of preparation of this report, the patient has completed 12 months of speech and auditory rehabilitation and is able to recognize $75 \%$ of sentences without lip-reading in open set tasks. The patient's performance in sentence recognition with lip-reading before and after $\mathrm{CI}$ according to the proposed speech activity, either in open or closed set tasks, is shown in - Fig. 1. The patient's performance in sentence recognition without lip-reading, either in closed or open set tasks, is shown in - Fig. 2. The rate of $100 \%$ means all sentences were recognized. So far, the patient has attended 15 sessions of speech therapy based on auditory-verbal therapy, which is aimed at the development

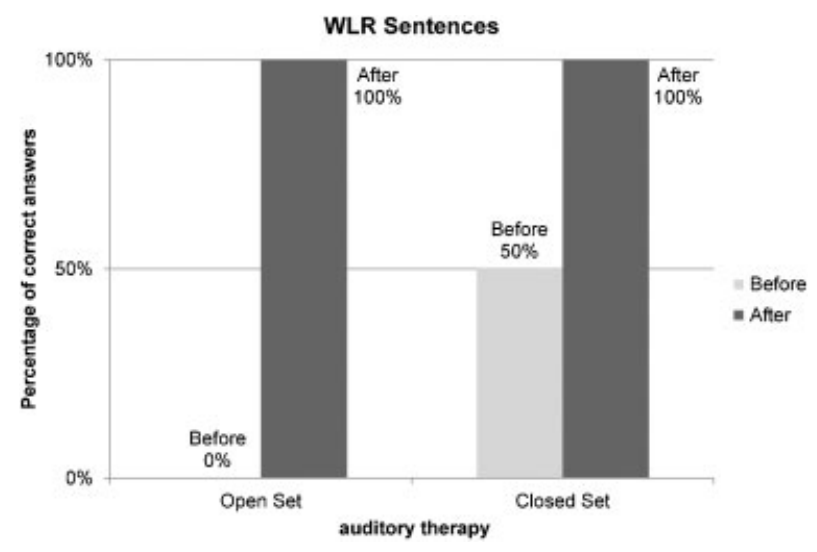

Fig. 1 Percentage of correct recognition of sentences with lip-reading (WLR) before and after cochlear implant. WLR: visual support from mime and lip-reading. Open set: understanding speech activity without agreeing with the examiner on what to say.

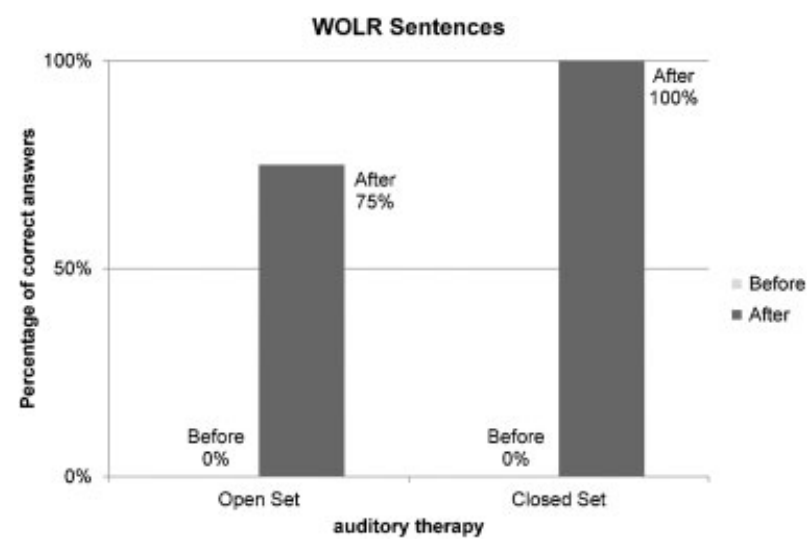

Fig. 2 Percentage of correct recognition of sentences without lipreading (WOLR) before and after cochlear implant. WOLR: without visual support from mime and lip-reading. Open set: understanding speech activity without agreeing with the examiner on what to say. Closed set: understanding speech activity after agreeing with the examiner on what to say.

of auditory and language skills according to Auditory Verbal International. $^{8,9}$

The deprivation of sight and hearing can cause drastic changes in the person's access to information and development. Patients with hearing loss who use bilateral personal sound amplification device or $\mathrm{CI}$, according to the individual selection criteria, must be followed in an auditory (re)habilitation program designed to develop or restore the ability of auditory perception with activities that stimulate auditory skills, among them auditory comprehension. 4,5,10-13

Auditory rehabilitation reduces the difficulties arising from hearing loss that restrict participation in social activities and that consequently result in a poorer quality of life. ${ }^{13}$ It is noteworthy that the auditory-verbal therapy focus on rehabilitation benefits individuals with or without genetic syndromes, blindness, and low vision. ${ }^{13-15}$

Patients with progressive hearing loss seem to respond better to $\mathrm{CI}$ than patients with sudden deafness as to the duration of hearing loss. ${ }^{10-12}$ Because our patient had visual 
and hearing loss, rehabilitation treatment was imperative, and $\mathrm{CI}$ has proven to be the best therapeutic option to improve the patient's quality of life thus far.

\section{References}

1 Harboyan G, Mamo J, Karam F, Karam FA. Congenital corneal dystrophy. Progressive sensorineural deafness in a family. Arch Ophthalmol 1971;85:27-32

2 Judisch GF, Maumenee IH. Clinical differentiation of recessive congenital hereditary endothelial dystrophy and dominant hereditary endothelial dystrophy. Am J Ophthalmol 1978;85(5 Pt 1): 606-612

3 Cawthorne TE, Hinchcliffe R. Familial perceptive deafness. Pract Oto-rhino-laryng 1957;19:69-83

4 Chiari BM, Bragatto EL, Nishihata R, Carvalho CAF. Perspectivas da atuação fonoaudiológica diante do diagnóstico e prognóstico da surdocegueira. Distur Comun São Paulo 2006;18(3):371-382

5 Puga ACS, Nogueira AHH, Félix TM, Kwitko S. Congenital corneal dystrophy and progressive sensorineural hearing loss (Harboyan syndrome). (Letter) Am J Med Genet 1998;80:177-179

6 Paparella MM, Sugiura S, Hoshino T. Familial progressive sensorineural deafness. Arch Otolaryngol 1969;90:44-51

7 Schuknecht HF. Auditory and cytocochlear correlates of inner ear disorders. Otolaryngol Head Neck Surg 1994;110:530-538
8 Easterbrooks SR, O'Rouke C. Gender differences in response to auditory verbal intervention in children who are deaf or hard of hearing. Am Ann Deaf 2001;146:309-319

9 Eriks-Brophy A. Outcomes of auditory-verbal therapy: a review of the evidence and a call for action. The Volta Review 2003;104:21-53

10 Goffi-Gomez MVS, Guedes MC, Sant'ana SBG, et al. Critérios de seleção e avaliação médica e audiológica dos candidatos ao implante coclear: HC-FMUSP. Arq Otorrinolaringol 2004;8: 303-323

11 Guedes MC, Brito RV, Goffi-Gomes MVS, et al. Telemetria de resposta neural intra-operatória em usuários de implante coclear. Rev Bras Otorrinol 2005;71:660-667

12 Bento RF, Brito Neto RV, Castilho AM, Goffi-Gomez VM, Giorgi SB, Guedes MC. Resultados auditivos com implante coclear multicanal em pacientes submetidos a cirurgia no Hospital das Clínicas da Faculdade de Medicina da Universidade de São Paulo. Rev Bras Otorrinolaringol (Engl Ed) 2004;70:632-637

13 Freire KGM. Reabilitação de deficientes auditivos adultos. In: Bevilacqua MC, Martinez MAN, Balen SA, Pupo AC, Reis ACMB, Frota S, eds. Tratado de Audiologia. Santos: Ed Santos; 2012:761774

14 Boothroyd A. Adult aural rehabilitation: what is it and does it work? Trends in Amplification 2007;(6):63-71

15 Política Nacional de Atenção à Saúde Auditiva. Available at: http:// dtr2001.saude.gov.br/sas/PORTARIAS/Port2004/PT-587.htm. Accessed July 22, 2013 